\title{
我国数字创意技术发展现状与展望
}

\author{
邓否 ${ }^{1}$, 王妙辉 $^{1}$, 范雷东 ${ }^{1}$, 袁圆 ${ }^{1}$, 王振中 ${ }^{2}$ \\ （1. 深圳大学电子与信息工程学院，深圳 518060；2. 中央广播电视总台技术管理中心，北京 100020）
}

\begin{abstract}
摘要：数字创意产业作为国家 “十三五” 规划确定的战略性新兴产业之一, 以全球数字化和网络化为背景, 以文化创意、内 容生产、版权利用为发展核心, 通过融合渗透带动周边产业集群的发展。相关产业发展壮大的基础性支撑在于数字创意技术 与创新设计, 而这又是我国产业发展的薄弱环节, 且面临未来发展路径的缺失状态。本文结合产业数据调研结果, 系统梳理 了我国数字创意技术中的使能技术、应用技术和终端设备技术的发展现状, 总结了超高清视频、三维声、虚拟现实/ 增强现 实 (VR/AR) 、数字内容生产和创新设计软件等具体方向存在的问题与短板。研究表明, 设立数字创意技术国家科技重大专 项, 建设数字创意技术国家工程研究中心, 提高数字创意技术人才培养质量, 积极参与国际合作并扩大中国文化的影响力, 将有力推动我国数字创意技术的中长期发展。
\end{abstract}

关键词: 数字创意; 文化创意; 创新设计；超高清视频；虚拟现实

中图分类号：TP3 文献标识码：A

\section{Development Status and Prospects of Digital Creative Technology in China}

\author{
Deng Lei ${ }^{1}$, Wang Miaohui ${ }^{1}$, Fan Leidong ${ }^{1}$, Yuan Yuan ${ }^{1}$, Wang Zhenzhong ${ }^{2}$ \\ (1. College of Electronics and Information Engineering, Shenzhen University, Shenzhen 518060, China; \\ 2. Technical Management Center, China Media Group, Beijing 100020, China)
}

\begin{abstract}
As one of the strategic emerging industries identified in the 13th Five-Year Plan of China, digital creative industry lives in the digital and networked era; it focuses on culture, content, and copyright, and drives the development of surrounding industries through integration and penetration. The key to developing the digital creative industry is digital creative technology and creative design, where, however, China has weaknesses and does not have a clear development path. In this paper, by analyzing industry data, we systematically describe the current development status of digital creative technology in China, including enabling technology, application technology, and end-user device technology, and summarize the problems and weaknesses of China in ultra-high definition (UHD) video, 3D audio, virtual/augmented reality, and software for digital content generation and creative design. Our investigation suggests that it will promote the development of China's digital creative technology by: (1) establishing national science and technology key projects and national engineering research centers on digital creative technology, (2) improving the quality of talents on digital creative technology, and (3) actively participating international cooperation and increasing the influence of Chinese culture.
\end{abstract}

Keywords: digital creativity; cultural creativity; creative design; ultra-high definition (UHD) video; virtual reality

收稿日期 : 2019-12-23; 修回日期 : 2020-02-27

通讯作者: 王振中, 中央广播电视总台教授级高级工程师, 研究方向为数字创意技术; E-mail: wangzhenzhong@cctv.com

资助项目：中国工程院咨询项目 “新兴产业发展战略研究（2035）”(2018-ZD-12)

本刊网址：www.engineering.org.cn/ch/journal/sscae 


\section{一、前言}

数字创意产业是在全球数字化和网络化的背 景下, 以数字创意技术与创新设计为基础支撑, 以 文化创意、内容生产、版权利用为发展核心，通 过融合渗透带动周边产业领域发展的新兴产业集群 $[1,2]$ 。数字创意产业覆盖众多细分领域, 包括超高 清产业、虚拟现实/增强现实 (VR/AR) 产业、设 计业、影视传媒业、动漫游戏业、数字出版业、人 居环境设计业、文化与博物馆业、时尚服饰业、玩 具业、体育健康业、旅游业等。数字创意产业将现 代信息技术与文化艺术领域高度融合, 促进文化艺 术创新和商业模式创新, 推动传统制造业、文化创 意产业和设计服务业的相互渗透和变革, 从而创造 新的经济增长点和业态模式。

2016 年，《“十三五”国家战略性新兴产业发展 规划》明确了数字创意产业作为国家战略性新兴产 业之一的关键性地位, 提出到 2020 年数字创意产 业发展成为重点培育的 5 个产值规模达 10 万亿元 的新支柱产业之一。在数字创意产业中, 数字创意 技术提供基础支撑能力, 体现了数字化环境中产生 的信息与传播的所有形式, 同时也代表了新一代信 息技术和创意产业深度融合与应用的所有形式。数 字创意技术分为 3 类：使能技术、应用技术和终端 设备技术 [2]。

使能技术是指通用的基础信息技术，包括人工 智能 $(\mathrm{AI})$ 、大数据、云计算、人机交互、类人视听 觉技术、空间及情感等感知技术、物联网与第五代 移动通信 $(5 \mathrm{G})$ 等网络技术。这些技术是各行业进 行数字化建设的前提条件, 也为数字创意行业自身 应用的实施提供支撑。应用技术是指各专业技术领 域实现层面的技术，如综合广播宽带（IBB）技术、 数字内容加工处理软件、家庭娱乐产品软件、动漫 游戏引擎技术、交互娱乐引擎开发、数字艺术呈现 技术、广播影视融合媒体直播技术以及文化资源数 字化处理技术等。应用技术是数字创意产业各专业 领域提升内容质量和创新服务模式的核心工具, 只 有在应用技术层面形成突破, 才能实现内容产品质 量提升。终端设备是用户进行内容消费的承载对象, 包括硬件及其装载的应用软件, 如 $4 \mathrm{~K} / 8 \mathrm{~K}$ 超高清 电视机、裸眼 3D 电视机、支持三维声 (3D Audio) 的沉浸式音频设备、VR/AR 设备、数据手套及游戏
控制器等感知终端、影视摄录设备、激光放映设备、 全息投影设备等。终端设备是改善用户体验的直接 手段, 其先进程度直接决定数字内容的呈现方式和 观众的使用感受。

目前已有论文针对其中某些技术领域进行了相 关阐述，如 AI 技术 [3]、大数据技术 [4]、云计算 技术 [5]、超高清电视技术 [6]、VR 技术 [7] 等。但 从已有论文来看, 有关我国数字创意技术领域的系 统梳理和全面阐述仍属空白。本文选取关键技术子 领域, 详细介绍我国数字创意技术领域的发展情 况。具体而言, 将以 AI、大数据、云计算、未来 网络技术为主介绍数字创意使能技术, 以综合广 播宽带、数字内容加工处理、动漫游戏引擎以及 文化资源数字化处理技术为主介绍数字创意应用 技术，以 $4 \mathrm{~K} / 8 \mathrm{~K}$ 超高清视频、三维声、VR/AR、 全息投影技术为主介绍数字创意终端设备技术。 整体研究架构如图 1 所示。

\section{二、数字创意技术的发展现状}

\section{（一）使能技术}

\section{AI 技术}

$\mathrm{AI}$ 是研究、开发用于模拟和延伸扩展人类智 能的理论、方法、技术以及应用系统的技术科学 [8]。最终目标是了解人脑智能的实质, 研制出一种 新的能以与人类智能相似的方式做出反应的智能机 器。 $\mathrm{AI}$ 的主要发展阶段分为运算智能、感知智能和 认知智能, 目前处于由感知智能向认知智能突破的 阶段。 $\mathrm{AI}$ 已经成为国际竞争的新领域, 是引领未来 的战略性技术, 越来越多的国家将发展 $\mathrm{AI}$ 作为提 升国家竞争力、维护国家利益的重大战略, 围绕核 心技术、顶尖人才、标准规范等强化部署, 力图在 新一轮国际科技竞争中掌握主导权。美国和中国在 计算机视觉、自然语言处理、智能机器人等技术方 向都处于领先水平。根据前瞻产业研究院发布的数 据 [9], 2019 年中国 AI 市场规模约为 644 亿元（见 表 1), 未来发展前景广阔。

我国已将 $\mathrm{AI}$ 技术应用到数字创意产业。例如, 在新闻创作方面, 腾讯公司推出的 Dreamwriter 可根据文本生成规则和章法自动地生成新闻报道。 在设计智能方面, 阿里巴巴公司开发的 “鹿班” $\mathrm{AI}$ 设计师软件, 每秒种能完成 8000 张海报设计, 


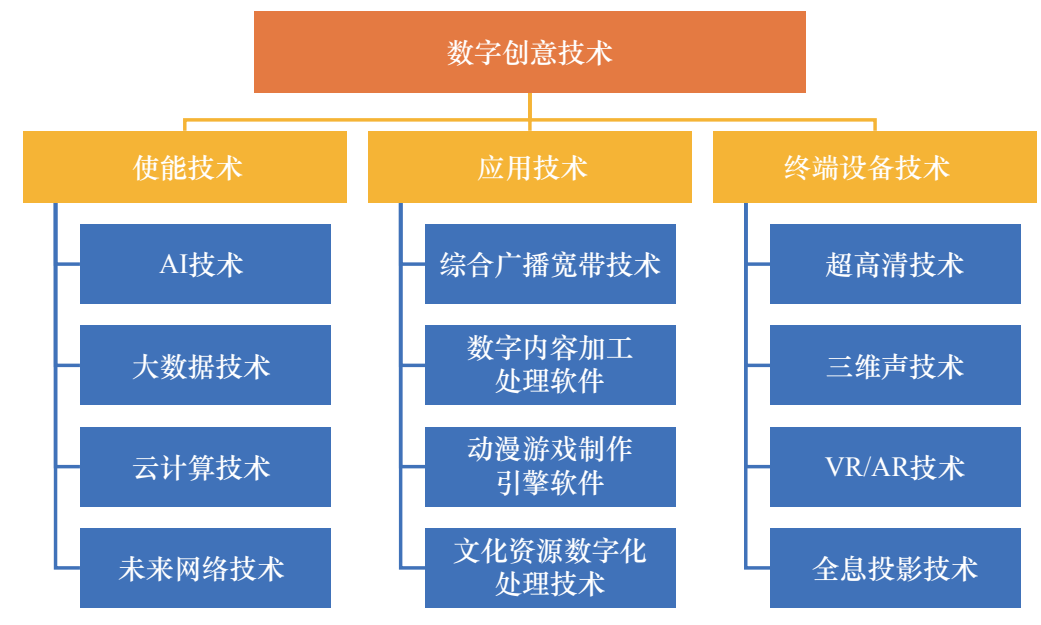

图 1 数字创意技术分类图

表 1 中国 AI、大数据及云计算市场规模 [10 12]

\begin{tabular}{lcccccc}
\hline 技术领域 & 规模 & 2015 年 & 2016 年 & 2017 年 & 2018 年 & 2019 年 \\
\hline $\mathrm{AI}$ & 市场规模/亿元 & 112 & 142 & 237 & 416 & 644 \\
& 同比增长 $/ \%$ & - & 26.8 & 66.9 & 75.5 & 54.9 \\
大数据 & 市场规模/亿元 & 2940 & 3700 & 4800 & 6170 & 8080 \\
& 同比增长 $/ \%$ & - & 25.9 & 29.7 & 28.5 & 31.0 \\
\multirow{2}{*}{ 云计算 } & 市场规模/亿元 & 387 & 510 & 692 & 963 & 1291 \\
& 同比增长 $/ \%$ & - & 31.8 & 35.7 & 39.2 & 34.1 \\
\hline
\end{tabular}

1 天可以制作 4000 万张海报。随着新一代 AI 技术 的发展, $\mathrm{AI}$ 在未来数字创意产业中将会扮演更为 重要的角色。

\section{2. 大数据技术}

大数据是一种凭借强大的存储和运算能力对大 量数据进行计算分析从而指导预测的技术。大数据 具有 “ $4 \mathrm{~V}$ ” 特征: 规模 (volume)、类型 (variety)、 速度（velocity）、价值（value）[10]。大数据涉及 的热门领域应用研究包括电子商务大数据、社交网 络大数据、交通大数据和视频大数据等。目前, 大 数据已在行业解决方案、计算分析服务、存储服务 等领域发挥着重大作用, 同时在工业应用层面保持 高速发展。根据中商产业研究院对大数据相关企业 的调研测算 [11], 2019 年我国大数据市场产值约为 8080 亿元，同比增长超过 30\%（见表 1)。

近五千年的历史文明使我国拥有丰富的艺术 品、文物、非物质文化遗产等文化资源以及大量的 公共图书馆、博物馆、美术馆等公共文化机构。在 对这些文化资源进行数字化处理的过程中, 产生了 大量数据, 在形式上表现为文本、图片、语音、视
频等。有效地存储、检索和应用这些文化数据都需 要使用相关大数据技术。目前, 我国已经搭建了多 个文化艺术领域的大数据平台, 如甘肃省的华夏文 化资源云平台、北京市的博物馆大数据平台以及故 宫的数字文物库等。

\section{3. 云计算技术}

云计算是一种通过网络以按需、易扩展的方式 获得所需服务 (硬件、平台、软件等) 的计算模式。 以基础设施即服务 ( IaaS)、平台即服务 ( PaaS ) 和 软件即服务 (SaaS ) 为代表的全球公有云市场规模 在 2018 年达到 9624.6 亿元, 增速为 $23 \%$ 。前几年 增长最快的部分是 IaaS, 而 SaaS 虽然增长速度减 缓, 但预计未来仍然将占据全球公共云市场的最大 份额 [12]。美国是云计算领域的早期创新者, 目前 仍占据着全球云服务市场的主导地位。我国的云计 算服务起步较晚, 虽然全球市场占比较小, 但增长 势头明显。根据《中国云计算产业发展白皮书》的 数据 [12], 2019 年中国云计算市场规模达到千亿元 水平，同比增长约 $34.1 \%$ （见表 1)。

云计算为数字创意提供计算和存储平台。目 
前, 我国已经出现了基于云计算的数字创意产品和 服务, 如阿里云提供的云剪辑功能, 可在云端进行 剪切拼接、混音、字幕、图片叠加、遮标、转场特 效等一系列操作, 解决了视频创作者本地计算能力 不足的问题。

\section{4. 未来网络技术}

$5 \mathrm{G}$ 是未来网络技术的代表。2019年发布的 《全球 $5 \mathrm{G}$ 竞赛》报告显示, 中国和美国在 $5 \mathrm{G}$ 商 业部署计划数量上并列世界首位 [13]。在 $5 \mathrm{G}$ 标准 的核心专利声明数量方面 (见图 2), 我国 3 家主 要企业的专利声明总量为 3542 件, 占世界总声明 量的 $30.3 \%$ 。这表明我国 $5 \mathrm{G}$ 技术研发处于全球领 先水平。

$5 \mathrm{G}$ 技术丰富了视频传输手段, 为超高清产业、 $\mathrm{VR}$ 产业发展提供了重要的传输能力支撑。2019 年 1 月, 中国移动通信集团有限公司联合中央广播电 视总台、华为技术有限公司在广东深圳成功开展了 基于 $5 \mathrm{G}$ 网络的 $4 \mathrm{~K}$ 电视信号传输测试, 将 $4 \mathrm{~K}$ 超高 清北京景观信号传送至位于深圳分会场的 $4 \mathrm{~K}$ 超高 清转播车。

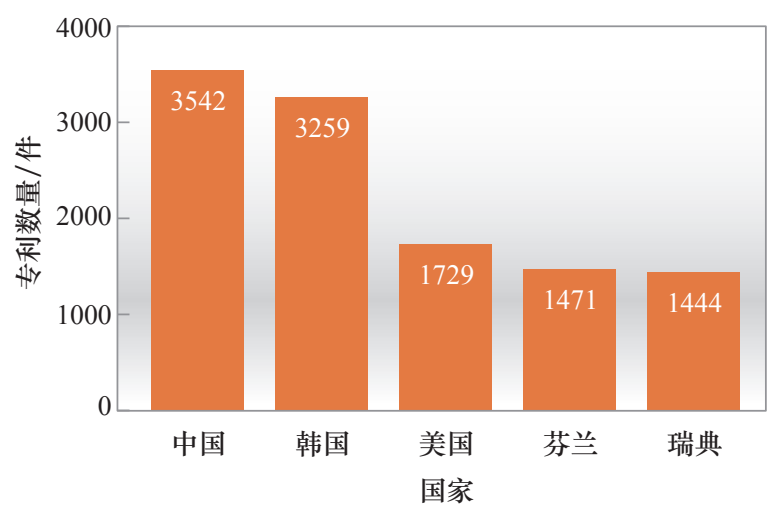

图 2 主要国家在欧洲电信标准化协会声明的 $5 \mathrm{G}$ 标准必要 专利数量（截至 2018 年 12 月 28 日） [14]

\section{（二）应用技术}

\section{1. 综合广播宽带技术}

IBB 技术综合了广播与宽带两种网络技术的优 势, 将电视节目和应用通过广播网和互联网两条传 输通道下发, 在用户的电视和移动终端上以一体化 的方式进行呈现, 从而为观众提供广播和交互的综 合体验 [15]。IBB 技术的核心理念是利用广播通道 和互联网通道来展现同一个电视节目, 观众在通过 广电网络收看传统电视节目的同时, 由电视台或
第三方应用服务提供商制作的业务相关类应用经由 宽带网通道传递给用户电视, 并以页面的形式与电 视节目视频画面共同呈现给观众; 还可通过设备关 联来将应用推送至手机、平板电脑、笔记本电脑等 移动终端 (见图 3)。目前 IBB 系统的标准化工作 以及应用产品已经在全球多个国家和地区部署, 而 我国正在进行 IBB 标准的制定工作。

\section{2. 数字内容加工处理软件}

数字内容加工处理是指将图片、文字、视频、 音频等信息内容运用数字化技术进行加工处理并 整合应用的服务, 主要分为两个层次的关键技术。 第一个层面是数字内容增强技术 [16]。例如, 百 度 $\mathrm{AI}$ 开发平台基于深度学习图像处理技术, 能对 质量较低的图片进行去雾、无损放大、对比度增强 等多种处理; 北京市商汤科技开发有限公司发布的 SenseAR 软件产品, 与 AR 技术结合可实现相机成 像的美体塑形功能。第二个层面是数字内容认知技 术 [17], 主要是指通过 AI 技术对图片和音视频等 数字内容进行初步理解, 通过适配文字解读或说明 的方式来帮助用户进行信息的智能检索或检测, 从 而显著节省人力物力。例如, 科大讯飞股份有限公 司发布的讯飞听见软件, 利用语音识别技术来实现 将音频转为文字的功能, 还可支持多国语言的音频 转换。

\section{3. 动漫游戏引擎软件}

在动漫和游戏领域内, 引擎是指可编辑动漫 游戏系统或者交互式实时图像应用程序的核心组 件。相关系统或组件为动漫游戏设计者提供编写 动漫游戏所需的各种工具, 旨在便捷快速地开发 动漫游戏产品, 而不必从底层算法开始产品设计。 引擎技术包括渲染引擎 (即 “渲染器”, 分为二维 图像引擎和三维图像引擎)、物理引擎、碰撞检测 系统、音效、脚本引擎、电脑动画、 $\mathrm{AI}$ 、网络引 擎以及场景管理等基本模块 [18]。目前在动漫游 戏引擎软件方面, 国内企业主要还是使用国外产 品 (如新加坡 Unity 技术公司的 Unity 实时内容开 发平台)。

国内企业自主研发或与国外企业合作开发了若 干引擎, 但技术水平与国外差距明显。随着国内企 业自主研发能力的提升, 致力于引擎技术本土化的 公司数量开始增多, 国内相关行业有望缩小与世界 级游戏开发水平的差距。 


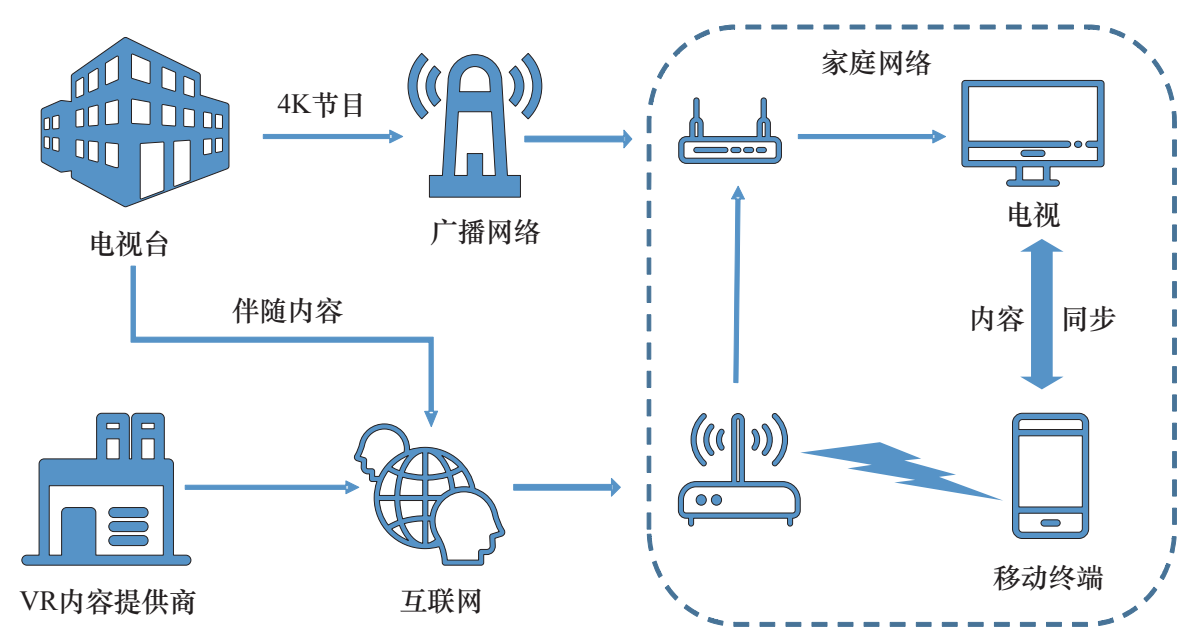

图 3 IBB 技术应用场景

\section{4. 文化资源数字化处理技术}

我国拥有丰富的文化资源, 这是我国数字创 意产业的基石和源泉。我国亟需对这些文化资源进 行数字化处理以更好地借助互联网技术来进行广 泛传播。我国文化资源数字化处理技术可追溯到 1998 年河南博物院建立的互联网网站。之后, 各地 博物馆普遍开始建设互联网网站, 数字技术应用于 文化资源的范围逐渐扩展。2001 年国家文物局启动 实施了 “文物调查及数据库管理系统建设” 项目, 统一开展全国博物馆的数字化工作。当前, VR、 直播、增材制造、手机移动客户端开发等新兴技术 的涌现, 进一步助力文化资源的推广传播, 发展形 成了 VR 展示、文物保护修复、博物馆管理、博物 馆教育等新兴应用。例如, 故宫借助《每日故宫》 移动客户端, 以日历的形式推出 “每天一件故宫藏 品”, 让全国观众随时随地欣赏故宫藏品; 故宫推 出了 “故宫博物院数字文物库” “全景故宫” “故宫 名画记” “数字多宝阁” 等众多数字化产品来拓展 故宫文物的影响力。

\section{（三）终端设备技术}

\section{1. 超高清技术}

视频是信息呈现和传播的主要载体，也是数字 创意产业的主要内容形式。当前, 视频技术正经历 从高清到 $4 \mathrm{~K}$ 超高清再到 $8 \mathrm{~K}$ 超高清的演进。4K 超 高清视频的分辨率为 $3840 \times 2160$, 是全高清视频 $1920 \times 1080$ 分辨率的 4 倍; $8 \mathrm{~K}$ 超高清视频的分辨
率为 $7680 \times 4320$, 是全高清视频分辨率的 16 倍 [6]。 超高清技术已经成为全球广播电视领域最为热门的 技术话题之一, 众多国家都在积极部署推进超高清 技术。

超高清视频设备的核心元器件主要包括感光 器件、存储芯片、编解码芯片、图像芯片、处理 器芯片和显示面板等。随着系统集成度不断提升, 存储芯片、解码芯片和处理器芯片已经综合为系 统级（SoC）芯片。2019 年国产芯片在国产电视 中开始应用, 超高清芯片受制于人的局面被打破。 目前, 国内企业在液晶面板和有机电激光显示 (OLED) 面板方面已经拥有较多数量的自主知识 产权。

\section{2. 三维声技术}

音频技术经历了单声道音频、双声道立体声音 频、5.1 环绕声、7.1 环绕声、三维声 (3D Audio) 的发展历程。三维声是具有三维空间感、方位感的 声音, 能使听众在听音过程中定位到声源的位置和 方向、听出声源的移动轨迹, 从而获得身临其境的 沉浸感 [19]。目前, 三维声技术以国外方案为主, 如成熟的 MPEG-H、Dolby 等。2016 年中央电视台 和广播科学研究院等组织成立了三维声专题组, 完 成了《三维声（3D Audio）广播电视行业标准技术 征集》, 开始正式征集提案; 2017 年各提案方正式 提交最终提案, 三维声测试评估组完成了相关标准 的测试验证。目前, 我国的三维声技术仍处于研发 阶段, 三维声的编解码技术和前后处理技术有待突 
破，三维声的制作技术标准、音频质量评审制度仍 需进一步完善。

\section{VR/AR 技术}

VR 通过电脑或其他智能计算设备来模拟产生 三维空间的虚拟世界, 为用户提供关于视觉、听觉、 触觉等感官的模拟感受。一般认为, VR 技术只展 示虚拟世界 [7]。AR 是将真实世界信息和虚拟世界 信息无缝集成, 将原本在现实世界一定的时间空间 范围内很难体验到的实体信息（如视觉信息、声音、 味道、触觉等) 进行模拟仿真后再叠加, 从而将虚 拟信息应用到真实世界; 这种信息可被人类感官所 感知, 获得超越现实的感官体验 [20]。

在产业政策方面, 我国已将 VR 产业发展上升 到国家高度。目前, 有近 20 个省（市、区）布局 了虚拟现实产业。在数字创意消费服务供给的丰富 度方面, VR 应用在教育图书、影视、游戏娱乐、 政务展览等方向发展迅速。虚拟内容资源不断丰 富, 在央视春晚、国家庆典、国际会展等重大活动 中开展了 VR 直播, 具有较强体验性的优质内容初 具规模。在 VR 产业标准方面, VR 头戴式显示设 备的标准化工作已经立项。也要注意到, 目前主流 的 VR/AR 设备仍然以 Oculus、微软公司等国外产 商的产品为主, 国内在 VR/AR 设备制造技术方面 与其存在一定差距。

\section{4. 全息投影技术}

全息投影技术通过集合被拍摄者反射的光波来 重建物体的全部信息, 使得平面物体内容得以立体 显示; 在给人立体视觉享受的同时, 提供了从不同 角度来更直观地观察物体的功能 [21]。随着 AI 技术 的发展与完善, 采用全息投影技术的产品应用已经 风麼全球，运用全息影像技术构建的舞台立体投影 效果, 达到身临其境的立体体验, 使得内容表现形 式更加直观、丰富和新颖。例如, 2016 年国内采用 全息投影技术导演了一场高难度演出一一在西湖水 面上表演《天鹅湖》舞蹈, 现场效果让观众叹为观止。

\section{三、数字创意技术面临问题分析}

整体来看, 我国在超高清视频技术、三维声技 术、 VR/AR 技术、数字内容生产和创新设计软件 等数字创意技术领域的创新支撑相对不足, 与美国、 英国、日本、韩国等发达国家还有一定差距。

\section{（一）超高清视频技术}

我国超高清视频技术仍然处于发展初期, 在取 得一定成绩的同时，仍面临诸多挑战。

1. 超高清技术攻关和摄像机等基础器件的研发 投入不足

摄像机是摄取超高清视频内容必不可少的关键 设备, 目前我国摄像机工业在基础器件研发和整机 集成方面只能进入中低端市场, 广播电视等高端领 域应用产品仍全部依赖进口。随着 $4 \mathrm{~K} 、 8 \mathrm{~K}$ 超高清 时代的来临, 我国面临更加严峻的技术攻关形势和 高端人才流失的挑战, 需要研发资源的持续投入。

\section{2. 行业测试标准规范缺乏}

已颁布的国家、行业标准集中在信源编解码和 终端接收显示等领域, 但包括信道、接口、安全、应 用在内的端到端超高清视频标准体系尚未完全建立。 相关技术标准的不统一和认证规范的缺失, 导致国 内现有的超高清视频技术参差不齐, 不利于形成观 众的优质体验, 影响产业未来的普及度和健康发展。

3. 产业重点专利布局滞后

在超高清方面, 我国尚未形成支持产业发展的 专利布局, 电视厂商主要采用国际成熟技术, 支付 专利费的现象在短时间内难以缓解, 存在着产业经 济利益继续受损的隐患。

\section{4. 内容源头供给严重缺乏}

严格符合 $4 \mathrm{~K} 、 8 \mathrm{~K}$ 标准的视频内容整体偏少, 成为了制约超高清视频产业发展的瓶颈。内容制作 成本高、产出回报周期长、版权无法完全保护等问 题, 极大降低了内容制作企业在超高清视频制作方 面的投入意愿。

\section{（二）三维声技术}

我国的三维音频技术仍处于初期研发阶段, 相 关产品所使用的技术仍然以国外为主, 较多彩用了 MPEG-H 和 Dolby 等技术。具体而言, 我国应组织 研发力量, 在三维音频技术中的编解码技术、前后 端处理技术、制作技术标准、质量评价制度等方面 进行提高和改善。

\section{(三) VR/AR 技术}

\section{1. 系统性研发能力不足}

国内在 VR/AR 领域片面追求芯片、传感器、 显示器件、光学器件等硬件的单一性能参数, 忽 
视了系统整体性能的提高。应对解决 VR/AR 产品 实际应用效果欠佳的现实需求, 我国仍需增加研 发投入，推动 $\mathrm{VR} / \mathrm{AR}$ 观看设备从大尺寸的头戴式 设备向小尺寸的平板、智能手机的渗透, 系统性 提升 VR/AR 使用体验。

\section{2. 优质内容不足}

国内的 VR/AR 产业仍以硬件生产为主, 2018 年 虚拟现实行业硬件收入占比达 70\% [1]。在内容应 用领域，尽管我国在央视春晚、国家庆典、国际会 展等重大活动中开展了 VR 直播, 但是优质内容的 规模仍有明显不足。优质内容是提升 VR/AR 用户 体验的核心，只有具备足够的优质内容，用户才有 消费 VR/AR 产品的意愿。面向教育、医疗、电子 商务、工业制作等重点行业领域的 VR/AR 应用研 发设计存在短板, 需要增加垂直行业的 VR/AR 内 容与应用投入。

\section{3. 产业生态链不完善}

国内的 VR/AR 企业以中小型企业为主, 其产 品大多是针对行业某些特定的软硬件环节应用需 求。我国尚不具备完整的 VR/AR 产业链, 应补齐 VR/AR 产业短板，构建包括硬件设备、软件应用、 垂直领域在内的完整的端到端产业链。

\section{（四）数字内容生产和创新设计软件领域}

在数字创意内容的生产和制作方面, 我国的数 字内容加工处理软件支撑不足, 大量的工业设计软 件依赖进口, 如 3D Studio Max、Maya、AutoCAD 等。 高端数字内容制作和创新设计的软件, 包括 VR 内 容制作、工业设计软件、动漫游戏制作引擎软件等, 国产贡献率普遍不高, 受进口软件的影响较为明显。 对国外数字内容生产加工软件技术的依赖对我国数 字创意产业的健康发展构成了掣肘。应加大研发力 度、实施奖励机制、组织相关技术企业对这一类软 件进行高质量地开发, 尽快自主研发出能够替代甚 至取代国外软件的产品成果。

\section{四、对策建议}

\section{（一）设立数字创意技术国家科技重大专项}

建议设立数字创意技术国家科技重大专项, 在 数字创意技术领域加快追赶国际技术和产业的步
伐。加大关键核心技术、关键元器件、高端产品、 创新应用、软件平台与工具的研发力度, 解决数字 创意技术重点领域中的关键核心技术短板和 “卡脖 子” 问题。通过重大专项的实施, 使我国在数字文 化创意技术装备、数字内容创新和创新设计等领域 形成具有国际竞争力的高新技术研发与创新体系, 支撑相关产业发展并在全球数字创意市场中发挥更 为重要的作用。

\section{（二）建设数字创意技术国家工程研究中心}

数字创意产业是高度交叉融合的产业, 也是典 型的高新技术产业, 但存在着自主创新能力不强、 系统集成能力不足、新技术应用广度和深度有待提 高等问题。建议组织具有较强研究开发和综合实力 的高校、科研机构和企业等, 共同参与数字创意技 术国家工程研究中心的建设, 在数字创意关键共性 技术、装备和智能设计系统方面进行工程化研究和 验证, 形成有利于技术创新、成果转化的机制。中 心应成为产业与科研之间的桥梁, 肩负促进我国数 字创意相关产业技术进步和核心竞争能力提高的重 要使命。

\section{（三）建设文化数字内容资源共享平台}

发展数字创意产业既能创造新的经济增长点, 也能大力传承我国优秀文化、促进中国文化走向世 界。建议构建跨部门、跨区域、跨行业的数字内容 资源大数据平台和共享平台, 开展全国范围内的文 化艺术品数字化建设, 并借助 VR/AR、超高清视频、 $5 \mathrm{G}$ 等新一代信息技术来深度开发相关文化艺术数 字产品。应积极参加国际合作交流, 传播我国优秀 文化, 吸收国外文化艺术精髓。所构建和开发的相 关数字化平台与产品以及参与国际合作的广泛度, 将极大地促进人类文化艺术知识的积累与传承。

\section{（四）提高数字创意技术人才培养质量}

数字创意产业涉及诸多新一代信息技术, 高新 技术元素富集。技术创新是推动数字创意产业发展 的重要基础力量, 而高端人才与实际需求不匹配。 建议探索设置数字创意技术专业, 发展相关学科, 实施一流数字创意技术专业建设示范项目, 保障数 字创意技术人才的培养数量和质量。 


\section{参考文献}

[1] 中国工程科技发展战略研究院. 2020中国战略性新兴产业发展 报告 [M]. 北京: 科学出版社, 2019.

Chinese Academy of Science and Technology for Development. Report on the development of China's strategic emerging industries (2020) [M]. Beijing: China Science Publishing \& Media Ltd., 2019.

[2] 潘云鹤, 丁文华, 孙守迁, 等. 数字创意产业发展重大行动计划 研究 [M]. 北京: 科学出版社, 2019.

Pan Y H, Ding W H, Sun S Q, et al. Major action plan for the development of digital creative industry $[\mathrm{M}]$. Beijing: China Science Publishing \& Media Ltd., 2019.

[3] Pan Y H. Heading toward artificial intelligence 2.0 [J]. Engineering, 2016, 2(4): 409-413.

[4] 李国杰, 程学旗. 大数据研究: 未来科技及经济社会发展的重大 战略领域一大数据的研究现状与科学思考 [J]. 中国科学院院 刊, 2012, 27(6): 647-657.

Li G J, Cheng X Q. Research status and scientific thinking of big data $[\mathrm{J}]$. Bulletin of the Chinese Academy of Sciences, 2012, 27(6): 647-657.

[5] 徐保民, 倪旭光. 云计算发展态势与关键技术进展 [J]. 中国科 学院院刊, 2015, 30(2): 170-180.

Xu B M, Ni X G. Development trend and key technical progress of cloud computing [J]. Bulletin of the Chinese Academy of Sciences, 2015, 30(2): 170-180.

[6] 惠慧, 朱林林, 何大治. 超高清电视发展趋势研究 [J]. 电视技术, 2015, 39(6): 22-26.

Hui H, Zhu L L, He D Z. Study on development trend of ultra high-definition television [J]. Audio Engineering, 2015, 39(6): 22-26.

[7] 赵沁平. 虚拟现实综述 [J]. 中国科学: 信息科学, 2009, 39(1): 2-46.

Zhao Q P. Survey of Virtual Reality [J]. Science in China Series F-Information Sciences, 2009, 39(1): 2-46.

[8] Russell S J, Norvig P. Artificial intelligence: A modern approach [M]. Malaysia: Pearson Education Limited, 2016.

[9] 前瞻产业研究院. 2019-2024年中国人工智能行业市场前瞻与 投资战略规划分析报告 [R]. 深圳: 前瞻产业研究院, 2019 .

Qianzhan Industry Research Institute. Market outlook and investment strategy planning of China's artificial intelligence industry (2019-2024) [R]. Shenzhen: Qianzhan Industry Research Institute, 2019.
[10] Dean J. Big data, data mining, and machine learning: Value creation for business leaders and practitioners [M]. Hoboken: John Wiley \& Sons, 2014.

[11] 中商产业研究院. 2019年中国大数据产业市场前景研究报告 [R]. 深圳: 中商产业研究院, 2019.

China Commerce Industry Research Institute. Market outlook of China's big data industry (2019) [R]. Shenzhen: China Commerce Industry Research Institute, 2019.

[12] 国务院发展研究中心国际技术经济研究所. 中国云计算产业发 展白皮书 [R]. 北京: 国务院发展研究中心国际技术经济研究 所, 2019.

Institute of International Technology and Economy, Development Research Center of the State Council. White book of China's cloud computing industry [R]. Beijing: Institute of International Technology and Economy, Development Research Center of the State Council, 2019.

[13] Abecassis D, Stewart J, Nickerson C. Global Race to 5G-Update [R]. Washington DC: CTIA, 2019.

[14] 中国信息通信研究院. 通信企业 $5 \mathrm{G}$ 标准必要专利声明量最新排 名(2018) [R]. 北京: 中国信息通信研究院, 2019.

China Academy of Information and Communications Technology. The latest ranking on the number of required patents for $5 \mathrm{G}$ standards in communications enterprises (2018) [R]. Beijing: China Academy of Information and Communications Technology, 2019

[15] Sotelo R, Joskowicz J, Rondán N. An integrated broadcastbroadband system that merges ISDB-T with HbbTV 2.0 [J]. IEEE Transactions on Broadcasting, 2018, 64(3): 709-720.

[16] Yu G, Sapiro G. Image enhancement and restoration [M]. Boston: Springer, 2014.

[17] Guo Y, Liu Y, Oerlemans A, et al. Deep learning for visual understanding: A review [J]. Neurocomputing, 2016, 187: 27-48.

[18] Gregory J. Game engine architecture [M]. Boca Raton: CRC Press, 2017.

[19] Herre J, Hilpert J, Kuntz A, et al. MPEG-H 3D audio-The new standard for coding of immersive spatial audio [J]. IEEE Journal of Selected Topics in Signal Processing, 2015, 9(5): 770-779.

[20] Azuma R T. A survey of augmented reality [J]. Presence: Teleoperators \& Virtual Environments, 1997, 6(4): 355-385.

[21] Caulfield H J. Handbook of optical holography [M]. Netherlands: Elsevier, 2012. 